\title{
A NEW CLASS OF INFINITE PRODUCTS, AND EULER'S TOTIENT
}

\author{
GEOFFREY B. CAMPBELL
}

Mathematics Research Section

Institute of Advanced Studies

School of Mathematical Sciences

The Australian National University

GPO Box 4, Canberra, Australia, 2601

(Received May 31, 1991 and in revised form September 2, 1993)

ABSTRACT. We introduce some new infinite products, the simplest being

$$
(1-y) \prod_{k=2}^{\infty} \prod_{j \varepsilon \phi_{k}}\left(1-y^{k} q^{\jmath}\right)^{1 / k}=\left(\frac{1-y}{1-q y}\right)^{1 /(1-q)},
$$

where $\phi_{k}$ is the set of positive integers less than and relatively prime to $k$, valid for $|y| \wedge|q y|$ both less than unity, with $q \neq 1$. The idea of a $q$-analogue for the Euler totient function is suggested.

KEY WORDS AND PHRASES. Combinatorial identities, partitions, arithmetic functions, convergence and divergence of infinite products, lattice points in large regions, applications of sieve methods, combinatorial enumeration problems, generating functions.

1980 AMS SUBJECT CLASSIFICATION CODES. 05A19, 05A17, 10K20, 40A20, 10J25, 10H32, $05 \mathrm{~A} 15$.

\section{INTRODUCTION.}

We introduce some new infinite product identities, the simplest being

$$
(1-y) \prod_{k=2}^{\infty} \prod_{j \varepsilon \phi_{k}}\left(1-y^{k} q^{j}\right)^{1 / k}=\left(\frac{1-y}{1-q y}\right)^{1 /(1-q)},
$$

where $\phi_{k}$ is the set of positive integers less than and relatively prime to $k$, with $|y|$ and $|q y|$ both less than unity, and $q \neq 1$. This ties in with a far-reaching idea in analysis and combinatorics, the notion of a $q$-analogue. This notion is to replace, where appropriate, the integer parameter $n$ by $\left(1-q^{n}\right) /(1-q), n$ ! by $\Pi_{m=1}^{n}\left(1-q^{n}\right) /(1-q)$, etc.. This is most familiar in the study of vector spaces over finite fields and the theory of hypergeometric series. Here it emerges in connection with a class of infinite products with fractional (and in general, arbitrarily small) exponents.

By Exercise 10, p.327 of [3] we have

$$
\prod_{n=1}^{\infty}\left(1-x^{n}\right)^{\mu(n) / n}=e^{-x}, \quad|x|<1
$$

If $x$ is replaced by $y^{m}$ and the product operator $\Pi_{m=1}^{\infty}$ is applied to both sides, we obtain 


$$
\prod_{k=1}^{\infty}\left(1-y^{k}\right)^{\phi(k) / k}=e^{-y /(1-y)}
$$

by rearranging the product on the left and applying the identity

$$
\frac{\phi(n)}{n}=\sum_{d \mid n} \frac{\mu(k)}{k}
$$

for Euler's totient $\phi(n)=\left|\phi_{n}\right|$. If we now take logarithms in (1.3) and expand, we discover that (1.3) is the generating function expressing of the familiar identity

$$
\sum_{k \mid n} \phi(k)=n
$$

the Möbius inversion of (1.4). Now a $q$-analogue of (1.5) is

where

$$
\sum_{k \mid n} \psi_{q, n}(k)=q \frac{q^{n}-1}{q-1}
$$

$$
\psi_{q}(k)=\psi_{q, n}(k)=\sum_{j \varepsilon \phi_{k}} q^{j n / k}
$$

In fact, the generating function expression of (1.6) that reduces to (1.3) for $q=1$ is (1.1).

It is not yet clear what properties (e.g., the multiplicativity) of $\phi$ have analogues for $\psi_{q}(k)$ with $q \neq 1$. As a curiosity, we note that differentiation of (1.6) followed by $q \rightarrow 1$ yields a Möbius inversion of the shallow identity $\sum_{\delta \in \phi_{n}} j=\frac{1}{2} n \phi(n)$.

A somewhat more impressive looking result than (1.1) is

$$
(1+y) \prod_{k=2}^{\infty} \prod_{j \varepsilon \phi_{k}}\left(1+y^{k} q^{\jmath}\right)^{1 / k}=\left(\frac{(1-q y)^{q}(1+y)}{(1-y)^{q}(1+q y)}\right)^{1 /\left(1-q^{2}\right)},
$$

valid for the same conditions as in (1.1). However, if each side of (1.8) is multiplied by the corresponding side of (1.1), what emerges is in fact a case of (1.1).

In the spirit of Euler we shall explore a variety of formal identities having the "style" of (1.1) while keeping details about domains of convergence and branching in the complex domain at a minimum. Our main result (of which (1.1) is a special case) is the Theorem of $\S 2$.

For infinite product expansions bearing some degree of similarity to those displayed here (including some with fractional exponents) see [2, pp.175-182], [4, p. 236, p. 343], and [6-9]. Reference [9] also examines the behavior of certain infinite products with fractional exponents as functions of a complex variable. [5] derives Euler products from results herein.

\section{THE MAIN THEOREM.}

Identities (1.1) and (1.8) are the cases $\left(a_{k}\right)=( \pm y)^{k} / k,\left(b_{k}\right)=k(\log q) / q$ of the

THEOREM. If $\left(a_{k}\right)$ and $\left(b_{k}\right)$ are sequences of functions chosen for the following series to converge then

where $S_{k}$ is defined by

$$
\sum_{k=1}^{\infty} a_{k} \frac{1-\exp \left(b_{k} q\right)}{1-\exp \left(b_{k} q / k\right)}=\sum_{k=1}^{\infty} S_{k}
$$

$$
S_{k}= \begin{cases}\sum_{j=1}^{\infty} a_{\jmath}, & k=1, \\ \sum_{j=1}^{\infty} a_{\jmath k} \sum_{h \varepsilon \phi_{k}} \exp \left(b_{j k} h q / k\right), & k>1, .\end{cases}
$$


where $\phi_{k}$ is the set of positive integers less than and relatively prime to $k$.

PROOF. If we associate the sequence $\left(c_{k}\right)=\exp \left(b_{k} q\right)$ with $\left(b_{k}\right)$ so that the left side of $(2.1)$ is expanded thus

$$
\begin{aligned}
a_{1} & +a_{2}\left(1+c_{2}^{1 / 2}\right) \\
& +a_{3}\left(1+c_{3}^{1 / 3}+c_{3}^{2 / 3}\right) \\
& +a_{4}\left(1+c_{4}^{1 / 4}+c_{4}^{2 / 4}+c_{4}^{3 / 4}\right) \\
& +a_{5}\left(1+c_{5}^{1 / 5}+c_{5}^{2 / 5}+c_{5}^{3 / 5}+c_{5}^{4 / 5}\right) \\
& +a_{6}\left(1+c_{6}^{1 / 6}+c_{6}^{2 / 6}+c_{6}^{3 / 6}+c_{6}^{4 / 6}+c_{6}^{5 / 6}\right) \\
& +\cdots,
\end{aligned}
$$

we see that under certain conditions the terms rearrange into

$$
\sum_{j=1}^{\infty} a_{j}+\sum_{j=1}^{\infty} a_{2 j} c_{2 j}^{1 / 2}+\sum_{j=1}^{\infty} a_{3 j}\left(c_{3 j}^{1 / 3}+c_{3 j}^{2 / 3}\right)+\sum_{j=1}^{\infty} a_{4 j}\left(c_{4 j}^{1 / 4}+c_{4 j}^{3 / 4}\right)+\cdots
$$

where the indices of the $c$ terms are the countable set of Farey fractions in the interval $(0,1)$. Since $(2.5)$ is the same as the right side of (2.1) this completes the proof formally. The conditions which validate equivalence of (2.4) and (2.5) are trivially the criterion of the theorem that all the series of the theorem converge after $\left(a_{k}\right)$ and $\left(b_{k}\right)$ are chosen. End of proof.

\section{NEW PRODUCTS RELATED TO (1.1).}

(1.1) and (1.8) are representative of a large class of new infinite products which may be of interest in at least the Theory of Partitions. To abbreviate these products we define the product operator

$$
\prod_{j}=\prod_{k=2}^{\infty} \prod_{j \varepsilon \phi_{k}}
$$

The following identities are simply derived from the theorem or by combining (1.1) and (1.2) variously with $q, y$, chosen so that all denominators are defined.

$$
\begin{gathered}
\left(\frac{1+y}{1-y}\right)_{k} \prod_{j}\left(\frac{1+y^{k} q^{j}}{1-y^{k} q^{j}}\right)^{1 / k}=\left(\frac{(1-q y)^{2 q+1}(1+y)}{(1-y)^{2 q+1}(1+q y)}\right)^{1 /\left(1-q^{2}\right)} \\
(1+y)_{k} \prod_{j}\left(1-(-y)^{k} q^{j}\right)^{1 / k}=\left(\frac{1+y}{1+q y}\right)^{1 /(1-q)}, \\
\left(\frac{1+y}{1-y}\right)_{k} \prod_{j}\left(\frac{1-(-y)^{k} q^{j}}{1-y^{k} q^{j}} \frac{1+y^{k} q^{j}}{1+(-y)^{k} q^{j}}\right)^{1 / k}=\left(\frac{(1-q y)(1+y)}{(1-y)(1+q y)}\right)^{2 /(1-q)}, \\
{ }_{k} \prod_{j}\left(\frac{1+(-y)^{k} q^{j}}{1-(-y)^{k} q^{j}}\right)^{1 / k}=\left(\frac{(1-y)(1+q y)^{2 q+1}}{(1-q y)(1+y)^{2 q+1}}\right)^{1 /\left(1-q^{2}\right)} .
\end{gathered}
$$

There would seem to be many interesting questions arising from these infinite products, such as the behavior of the branching effects of the fractional indices on the left sides of the identities, or the combinatorial interpretations. In exactly which sense the infinite products are true as functions of the complex variables seems worthy of further investigation. We remark that their numerical validity for various real $q$ and $y$ may be easily checked with a small calculator. 


\section{SOME MORE GENERALIZED PRODUCTS.}

For $q$ and $y$ as previously, we evaluate

$$
\prod_{j}\left(1-y^{k} q^{\jmath}\right)^{\jmath^{m} / k^{m+1}}
$$

where $m$ is a positive integer. (1.1) relates to $m=0$ of (4.1). The following lemma is the case $\left(a_{k}\right)=y^{k} / k^{m},\left(b_{k}\right)=k(\log q) / q$ of the main theorem.

LEMMA. If $n \varepsilon \mathbb{C}, f(n, y)=\sum_{\lambda=1}^{\infty} y^{\lambda} / \lambda^{n},|y|<1$, and $\phi_{k}$ is as in $\S 1$ then

$$
f(n, y)+\sum_{k=2}^{\infty} \sum_{j \in \phi_{k}} f\left(n, q^{j} y^{k}\right) / k^{n}=(f(n, y)-f(n, q y)) /(1-q) .
$$

All series in (4.2) converge absolutely if both $|y|$ and $|q y|$ are less than unity with $q \neq 1$. If we differentiate (4.2) $m$ times with respect to $z=\log q$ then replace $n$ by $n+m$ we obtain

$$
\begin{aligned}
\sum_{k=2}^{\infty} \sum_{j \varepsilon \phi_{k}} j^{m} f\left(n, q^{j} y^{k}\right) / k^{n+m} & \\
& =f(n+m, y) g_{m}(q)-\sum_{k=0}^{m}\left(\begin{array}{c}
m \\
k
\end{array}\right) f(n+k, q y) g_{k+1}(q),
\end{aligned}
$$

where for non-negative integers $m$ and for $q \neq 1$ we define

$$
g_{m}(q)=\left.\frac{d^{m}}{d z^{m}}\left(1-e^{z}\right)^{-1}\right|_{z=\log q} .
$$

When $n=1$ in (4.3) we get that for each positive integer $m$, and for $q \neq 1$

$$
\begin{aligned}
& { }_{k} \prod_{j}\left(1-y^{k} q^{j}\right)^{j^{m} / k^{m+1}} \\
& \quad=\exp \left(-f(m+1, y) g_{m}(q)+\sum_{k=0}^{m}\left(\begin{array}{c}
m \\
k
\end{array}\right) f(1+k, q y) g_{k}(q)\right)
\end{aligned}
$$

For example, the cases of (4.5) with $m=1$ and $m=2$ are

$$
\begin{aligned}
& \left(1-y^{2} q\right)^{1 / 2^{2}}\left(1-y^{3} q\right)^{1 / 3^{2}}\left(1-y^{3} q^{2}\right)^{2 / 3^{2}}\left(1-y^{4} q\right)^{1 / 4^{2}}\left(1-y^{4} q^{3}\right)^{3 / 4^{2}} \ldots \\
& =(1-q y)^{-1 /(1-q)} \exp \left(q(1-q)^{-2}\left(\frac{q y-y}{1^{2}}+\frac{(q y)^{2}-y^{2}}{2^{2}}+\frac{(q y)^{3}-y^{3}}{3^{2}}+\cdots\right)\right), \\
& \left(1-y^{2} q\right)^{1 / 2^{3}}\left(1-y^{3} q\right)^{1 / 3^{3}}\left(1-y^{3} q^{2}\right)^{2^{2} / 3^{3}}\left(1-y^{4} q\right)^{1 / 4^{3}}\left(1-y^{4} q^{3}\right)^{3^{2} / 4^{3}} \ldots \\
& =(1-q y)^{-1 /(1-q)} \exp \left(\left(q+q^{2}\right)(1-q)^{-3}\left(\frac{q y-y}{1^{3}}+\frac{(q y)^{2}-y^{2}}{2^{3}}+\frac{(q y)^{3}-y^{3}}{3^{3}}+\cdots\right)\right. \\
& \left.+q(1-q)^{-2}\left(\frac{q y-y}{1^{2}}+\frac{(q y)^{2}-y^{2}}{2^{2}}+\frac{(q y)^{3}-y^{3}}{3^{2}}+\cdots\right)\right) .
\end{aligned}
$$

It follows trivially from (4.5) and the definition of the Bernoulli numbers that

$$
k \prod_{j}\left(1-y^{k}\right)^{\jmath^{m} / k^{m+1}}=\exp \left(\sum_{k=0}^{m}\left(\begin{array}{c}
m \\
k
\end{array}\right) B_{k} f(k, y)\right),
$$

for $|y|<1$ and any positive integer $m$. (4.8) can be also obtained from expanding (4.2) as 
power series in $\log q$ and equating coefficients of like powers.

\section{PRODUCTS INVOLVING PARTITION THEORETIC FUNCTIONS.}

The classes of infinite products derived in this paper may lead to new partition theoretic identities. For example, (1.1) may be said to enumerate certain weighted vector partitions, the principles of which are outlined in Chapter 12 of [1]. Elementary combinations of products such as (1.1) multiplied by itself with different arguments can build identities involving well known partition generating functions such as $(a ; q)_{n}=(1-a)(1-a q) \cdots\left(1-a q^{n-1}\right)$, (see for example, [1], [2]). However, the fractional indices in the products of this paper seem to bring a new slant to the known $q$-product ideas. For example, let us write (1.1) as a product tableaux:

$$
\begin{aligned}
\left(\frac{1-y}{1-q y}\right)^{1 /(1-q)} & \\
= & (1-y) \\
& \left(1-y^{2} q\right)^{1 / 2} \\
& \left(1-y^{3} q\right)^{1 / 3}\left(1-y^{3} q^{2}\right)^{1 / 3} \\
& \left(1-y^{4} q\right)^{1 / 4} \quad\left(1-y^{4} q^{3}\right)^{1 / 4} \\
& \left(1-y^{5} q\right)^{1 / 5}\left(1-y^{5} q^{2}\right)^{1 / 5}\left(1-y^{5} q^{3}\right)^{1 / 5}\left(1-y^{5} q^{4}\right)^{1 / 5} \\
& \left(1-y^{6} q\right)^{1 / 6} \quad\left(1-y^{6} q^{5}\right)^{1 / 6} \\
& \text { etc. }
\end{aligned}
$$

This tableaux excludes all terms $\left(1-y^{m} q^{n}\right)^{1 / m}$ such that $n$ is not relatively prime to $m$. However, these terms can be inserted into the tableaux when we take

$$
P(q, y) P\left(q^{2}, y^{2}\right)^{1 / 2} P\left(q^{3}, y^{3}\right)^{1 / 3} P\left(q^{4}, y^{4}\right)^{1 / 4} P\left(q^{5}, y^{5}\right)^{1 / 5} \ldots
$$

where $P(q, y)$ is the product (5.1). This leads to the identity

$$
\prod_{k=1}^{\infty}\left(\frac{1-y^{k}}{1-(q y)^{k}}\right)^{1 /\left(k-k q^{k}\right)}=\prod_{k=1}^{\infty}\left(y^{k}, q\right)_{k}^{1 / k}
$$

where $(y, q)_{n}=(1-y)(1-y q) \cdots\left(1-y q^{n-1}\right)$. Considering the partial products of (5.2) suggests that the radii of convergence of $q$ and $y$ in (5.3) is unity, where previously that of $q$ was dependent upon $1 / y$. This is evident from the terms $\left(1-y^{m} q^{m-1}\right)^{1 / m}$, in (5.1). Multiplying cases of (5.3) with $y q, y q^{2}, y q^{3}, \cdots$ substituted for $y$ gives

$$
\prod_{k=1}^{\infty}\left(1-y^{k}\right)^{1 /\left(k-k q^{k}\right)}=\prod_{k=1}^{\infty}\left(y^{k}, q\right)_{\infty}^{1 / k}
$$

\section{CONCLUSION.}

The results of this paper came only from considering cases of the Theorem in $\S 2$ where $\left(b_{k}\right)=k(\log q) / q$. However, other choices of $\left(b_{k}\right)$ may be of interest. In particular, the author has found Jacobi theta function transformations from the cases $\left(b_{k}\right)=k^{2}(\log q) / q$, and results for the arithmetic functions they generate. Numerous other products exist related to those stated herein. Some examples are (see Campbell [5])

$$
\begin{gathered}
(1-q y)_{k} \Pi_{j}\left(1-y^{\jmath} q^{k}\right)^{1 / \jmath}=(1-q y)^{1 /(1-q)}, \\
(1-y)(1-q y)_{k} \prod_{j}\left(1-y^{k} q^{\jmath}\right)^{1 / \jmath}\left(1-y^{\jmath} q^{k}\right)^{1 / k}=(1-y)^{1 /(1-q)},
\end{gathered}
$$


the former valid for $|q y|$ and $|q|$ both less than 1, the latter with $|q|$ and $|y|$ both less than 1 . (6.1) and (6.2) seem interesting for a variety of reasons. If $q=1-1 / n$ with $n$ chosen as a positive integer then the right sides of both identities reduce to a polynomial in $y$. The left sides of the identities enumcrate weighted vector partitions connected with the idea of lattice points in the $X Y$-plane being visible from other such lattice points. Also, the author has used products such as (1.1) to obtain new Euler products. (see [5]).

ACKNOWLEDGEMENT. The author is indebted to the Referee for his valuable comments and suggestions, and to Professor George E. Andrews for his encouragement and facilitation of this work.

\section{REFERENCES}

1. ANDREWS, G.E., The theory of partitions, vol. 2, Encyclopedia of Mathematics and its Applications, Addison-Wesley, Reading, Mass., 1976.

2. ANDREWS, G.E., q-Series: Ten Lectures, NSF-CBMS Regional Conference, 175-182, Arizona State University, 1985.

3. APOSTOL, T.M., Introduction to Analytic Number Theory, Springer-Verlag, New York, 1976.

4. BORWEIN, J. \& BORWEIN, P., Pi and the AGM, 236-343, John Wiley and Sons, New York, 1986.

5. CAMPBELL, G.B., Dirichlet summations and products over primes, Internat. J. Math. $\mathbb{G}$ Math. Sci. 16 (1993), 359-372.

6. FELD, J.M., The expansion of analytic functions in a generalized Lambert series, Annuls of Math. 33 (1932), 139-143.

7. FELD, J.M. \& NEWMAN, P., On the representation of analytic functions of analytic functions of several variables as infinite products, Bull. Amer. Math. Soc. 36 (2), (1930), 284-288.

8. RITT, J.F., Representation of analytic functions as infinite products, Math. Zeit. 32 (1930), 1-3.

9. STOLARSKY, K., Mapping properties, growth and uniqueness of Vieta (infinite cosine) products, Pac. J. Math. 89 (1980), 209-227. 


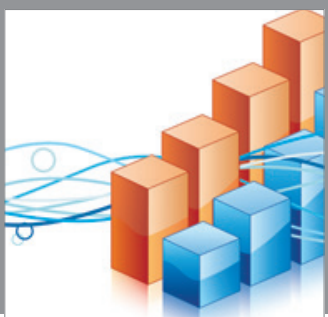

Advances in

Operations Research

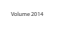

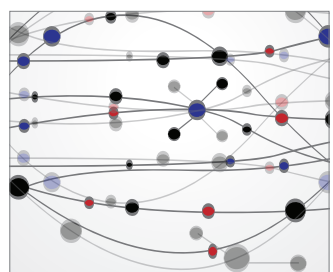

\section{The Scientific} World Journal
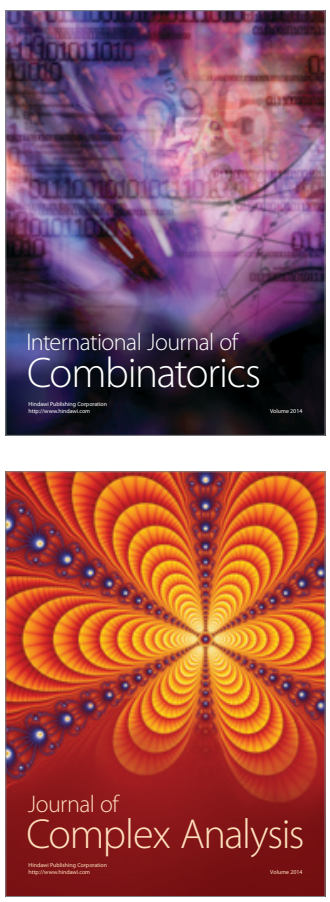

International Journal of

Mathematics and

Mathematical

Sciences
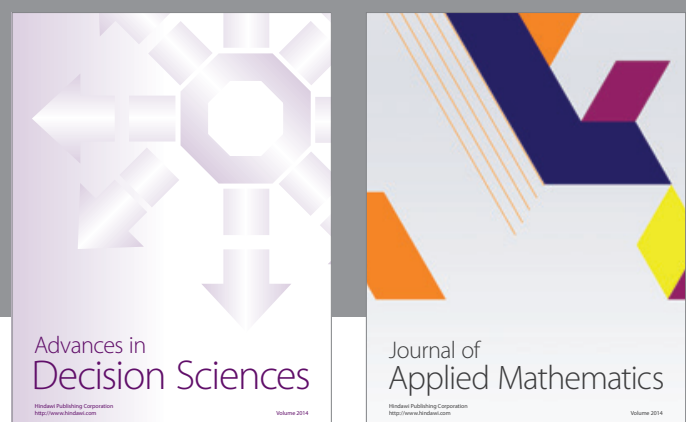

Journal of

Applied Mathematics
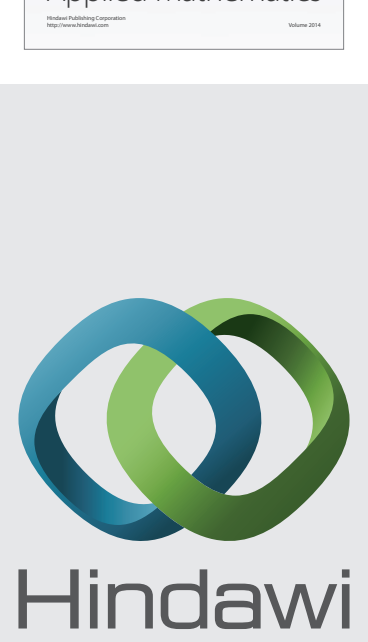

Submit your manuscripts at http://www.hindawi.com
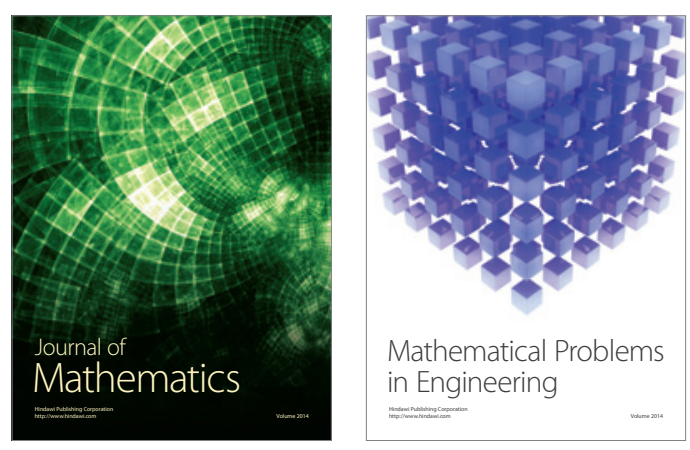

Mathematical Problems in Engineering
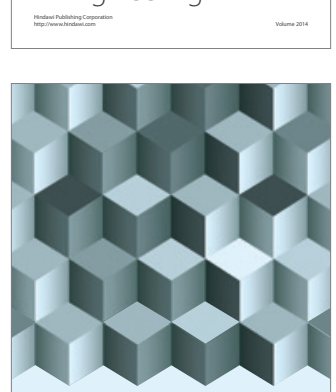

Journal of

Function Spaces
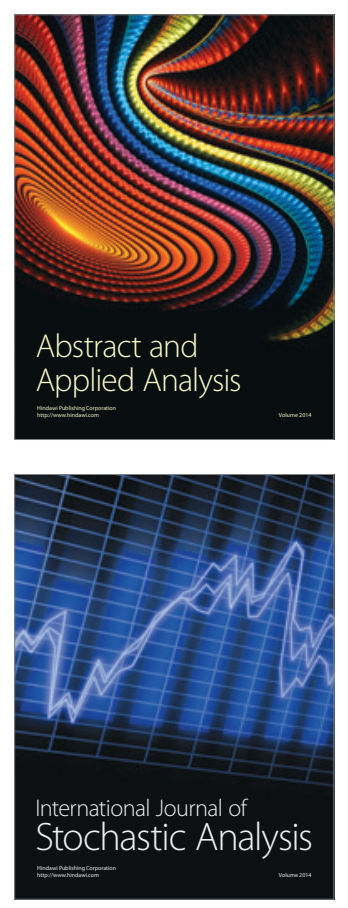

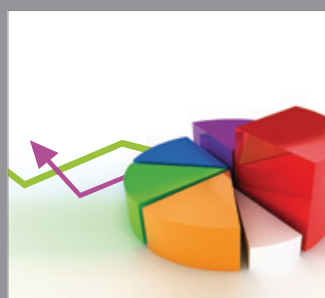

ournal of

Probability and Statistics

Promensencen
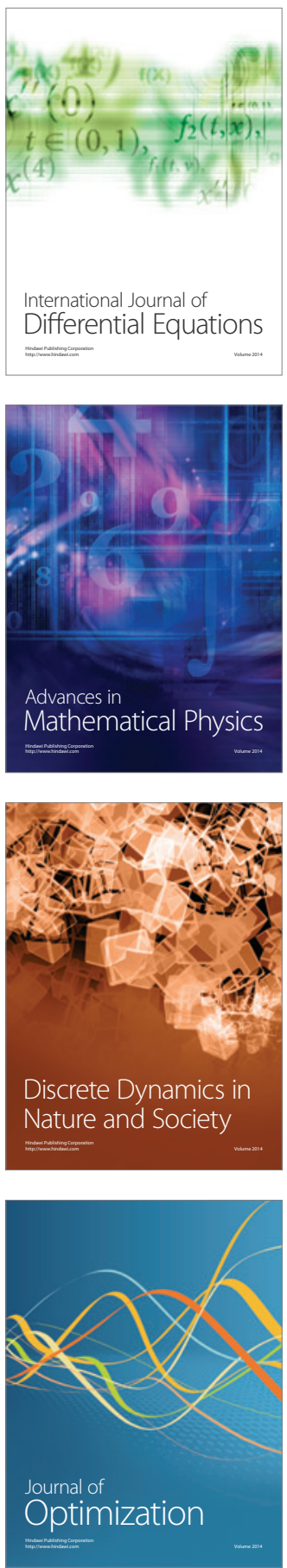